\title{
The Stable Bounded Theory: A Solution to Projecting the Total Fertility Rate in Mexico
}

\author{
By Javier González-Rosas * \\ Iliana Zárate-Gutiérrez"
}

Total fertility rate (TFR) is one of the demographic components that determine changes in population volume; thus, if we require population estimates in the future, it is absolutely necessary to project this component. However, projection of the TFR has two inherent problems: first, we have to determine that value where the TFR will stabilize in future, which is called the stabilizer value, and second, we have to determine the function which we are going to use to projecting the TFR. The stable bounded theory provides us solutions to these two inherent problems with solid scientific support. The first problem is solved by finding an estimate of the stabilizing value that depends on the observed data of the indicator. The second is solved by deducing a function that is stabilized precisely at the estimated stabilizer value. The main results of the article indicate that the TGF of Mexico will stabilize at 1.98 children per woman and that the function, although it belongs to the exponential family, is not a logistic function, which has traditionally been used to project the TFR. Other results indicate that the average TFR in Mexico in 2020 will be 2.16 children per woman; in 2030 it will be 2.07; in 2040 it will reach the figure of 2.03; and by 2050 , it will be 2.0 children per woman.

Keywords: Forecast, Fertility Specific, Stability, Total fertility rate.

\section{Introduction}

Population projections in Mexico usually have been elaborated by applying the method of demographic components, which is based on estimations of births, deaths, emigrants and immigrants, which effect changes in human populations. This method estimates births and deaths by projecting fertility and mortality using logistic functions. However, emigrants and immigrants have not been projected using mathematical models; rather, their projection is restricted only to assumptions about their future behavior.

The demographic components method provides information besides the population volume, such as the demographic dynamics of the country by predicting the future behavior of components like fertility, mortality and international migration. In Mexico, future fertility is projected first by the level and second by the structure. The level is obtained by projecting the TFR, which traditionally is estimated by adjusting a logistic function to the observed data. The use of the logistic function is justified because in Mexico the observed data of fertility through time follows a logistic pattern and is stabilized in the future. The

${ }^{*}$ Director of Socio economic Studies and International Migration, National Population Council, Mexico.

${ }^{\dagger}$ Deputy Director, Secretariat of the Interior, Mexico. 
problem is that there are at least two other functions that can also be adjusted to the observed data acceptably and that also stabilize in the future.

Nowadays, the value where fertility will stabilize is not estimated, but is fixed by an experts group convened by the United Nations. An age structure projection is done, first by defining the limit structure as the age composition of United Nations (UN) projections that correspond to the TFR fixed by the experts, and second by doing linear interpolations between limit structure and observed age composition (Partida-Bush 2008).

The Stable Bounded Theory solves the two inherent problems that are present when we want to forecast fertility, in other words the stability value problem and the problem of the function used for its projecting.

\section{Literature Review}

According to the data of the 2015 Revision of World Population Prospects, the TFR is now 2.5 children per woman globally. However, this global average masks wide regional differences. Africa continues being the region with the highest fertility, since its TFR is 4.7 children per woman, while Europe has the lowest fertility, with a TFR of 1.6 children per woman. Other regions, such as Asia, Latin America and the Caribbean, have a TFR of 2.2, closely followed by Oceania with 2.4 children per woman (United Nations 2015).

Fertility decline has been an important determinant of population aging. Consequently, fertility projecting has important implications for the age structure of future populations and the pace of population aging. The TFR is one of the key components in these population projections. Within a specific period, it is defined as the average number of children a woman would have if she survived through the end of the reproductive age, experiencing at each age the age-specific fertility rates of that period (Alkema et al. 2011).

In 2015, close to half of the world's population lived in countries where the period total fertility rates (TFR) were below the replacement level ${ }^{1}$. This included many middle- and lower-income countries in Asia and Latin America, such as Brazil, China, Iran, Thailand, Turkey and Vietnam. Some countries of Europe and East Asia even experienced levels of "lowest-low fertility" with a TFR falling below 1.3 children per woman. The current low fertility is sometimes viewed as temporary, caused by postponement of childbearing, economic uncertainty, a passing phase in the process of human development, or in the ongoing "gender revolution". In order to face the effects of these low fertility levels, governments will have to design new public policies (Sobotka et al. 2015).

In the UN, the population is projected using a cohort component method. According to this method, it is necessary to observe a population in a base year, as well as the future fertility rates, survival probabilities and migration net counts. All of these measures are taken in five-year age groups and according to gender. Given those inputs, the method assesses the population by both age and sex in year

\footnotetext{
${ }^{1}$ The replacement level means that a woman will have a daughter alive when her reproductive period finishes, what means, the woman will be replace to continuing the reproductive process.
} 
$\mathrm{t}+5$, adding to the population in year $t$ the births and net migration occurring during a 5-year period, minus the deaths that also occurred during the same period. Traditionally, the UN has produced deterministic population projections and punctual (that is, only one number). These point projections were supplemented with ranges based on different scenarios of demographic changes. In July 2014, the UN for the first time issued official probabilistic population projections, which quantify the uncertainty associated with demographic projections (Alkema et al. 2015).

An old question in economics that goes back at least until the time of Malthus is: how much higher should income per capita be if the fertility rate were to fall by a specified amount? Over the last half century, the consensus about the effects of fertility decline have changed from seeing them as strong to not very important, and recently back toward assigning them some significance. For an issue that has been studied for so long, and with such potential import, the evidence regarding the economic effects of fertility is rather weak. However, in 2013 Ashraf found that a reduction in fertility raises income per capita by an amount that some would consider economically significant. Although the effect is small relative to the vast gaps in income between developed and developing countries, the finding is a potential answer to the old question (Ashraf et al. 2013).

Europe has already completed its demographic transition. Demographic transition theory has been very useful for explaining global demographic trends during the 20th century, and still has strong predictive power when it comes to projecting future trends in countries with high fertility. However, it is not useful for predicting the future of fertility in Europe nowadays. The current notion of a second demographic transition is a useful way to describe a bundle of behavioral and normative changes that recently happened in Europe, but it has no predictive power. The problem is that there is not yet a useful theory to predict the future fertility level of post-demographic transition societies; we do not even know whether the trend will go up or down (Lutz 2006).

\section{Methodology}

\section{Data Used}

The sources of the data used in this paper are nine surveys with national representativeness regarding fertility and that have been conducted in Mexico during the period 1976-2015. Table 1 presents the specific and total fertility rates used in this article. The 1975 data were estimated by the Secretariat of Programming and Budget through the General Directorate of Statistics based on the Mexican Fertility Survey (MFS) of 1976. The data for 1978 were estimated by the Coordination of the National Family Planning Program based on the National Survey of Prevalence in the Use of Contraceptive Methods (NSPUCM) of 1979. The data of 1991, 1996 and 2005 were the authors' own calculations based on the National Surveys of the Demographic Dynamics (NSDD) of 1992, 1997 and 2006. The data of 2002 were also unique calculations based on the National Reproductive 
Health Survey (NRHS) of 2003. Finally, the data for 2009 and 2005 were estimated by the National Institute of Statistics and Geography (INEGI by its acronym in Spanish).

Table 1. Fertility Specific Rate per Age Group in Mexico, 1975-2014

\begin{tabular}{|c|c|c|c|c|c|c|c|c|}
\hline Year & $\mathbf{1 5 - 1 9}$ & $\mathbf{2 0 - 2 4}$ & $\mathbf{2 5 - 2 9}$ & $\mathbf{3 0 - 3 4}$ & $\mathbf{3 5 - 3 9}$ & $\mathbf{4 0 - 4 4}$ & $\mathbf{4 5 - 4 9}$ & TFR \\
\hline 1975 & 0.0989 & 0.2964 & 0.2941 & 0.2359 & 0.1840 & 0.0793 & 0.0165 & 6.03 \\
\hline 1978 & 0.0947 & 0.2392 & 0.2582 & 0.2226 & 0.1454 & 0.0594 & 0.0158 & 5.18 \\
\hline 1986 & 0.0840 & 0.2020 & 0.2030 & 0.1430 & 0.0970 & 0.0340 & 0.0050 & 3.84 \\
\hline 1991 & 0.0901 & 0.1952 & 0.1776 & 0.1264 & 0.0783 & 0.0281 & 0.0043 & 3.50 \\
\hline 1996 & 0.0769 & 0.1570 & 0.1523 & 0.1058 & 0.0543 & 0.0184 & 0.0022 & 2.83 \\
\hline 2002 & 0.0822 & 0.1415 & 0.1430 & 0.1027 & 0.0586 & 0.0187 & 0.0030 & 2.75 \\
\hline 2005 & 0.0645 & 0.1315 & 0.1277 & 0.0893 & 0.0424 & 0.0130 & 0.0026 & 2.36 \\
\hline 2009 & 0.0569 & 0.1318 & 0.1255 & 0.0950 & 0.0503 & 0.0167 & 0.0026 & 2.39 \\
\hline 2014 & 0.0625 & 0.1286 & 0.1182 & 0.0864 & 0.0459 & 0.0134 & 0.0020 & 2.29 \\
\hline
\end{tabular}

Source: 1975) Secretaría de Programación y Presupuesto, 1979, p. 138;

1978) Coordinación del Programa Nacional de Planificación Familiar, 1979, p. 27;

1986) Dirección General de Planificación Familiar, 1989, p. 36;

1991, 1996, 2005) Own calculations based on the NSDD of 1992, 1997 and 2006

2002) Own calculations based on the NRHS of 2003;

2009, 2014) INEGI, principales resultados de la Encuesta Intercensal de 2015

\section{Structure and Level of the TFR in Mexico at Last 40 Years}

In Mexico the available data indicate that the fertility structure has undergone very important changes during the period 1975-2014. In 1978, according to the NSPUCM, fertility structure was characterized by a curve with a late peak, since the greatest fertility was in the group 25-29 years old. In 1991, according to the 1992 NSDD, the fertility curve changed to an earlier peak, due to the greatest fertility occurring in the group 20-24 years old. For 2014, ICS's data of 2015 show that the fertility curve has an extended form, since the fertility rates in groups 20 24 and 25-29 years old are very similar (Figure 1).

Figure 1 also shows that the groups that contribute the most to the level of fertility (called majority groups) in all years are 20-24, 25-29, 30-34 and 35-39 years. The contribution of these groups to the level of fertility has not changed significantly. In 1978, the contribution of these groups was $83.6 \%$, in 1991 of $82.5 \%$ and in 2014 of $83.0 \%$. On the other hand, the groups that have a smaller contribution (called minority groups) are 15-19, 40-44 and 45-49 years. These groups accounted for 16.4\%, 17.5\% and 17.1\% in the years 1978, 1991 and 2014, respectively. 
Figure 1. Fertility Specific Rate per Age Group in Mexico, 1978, 1991 and 2014

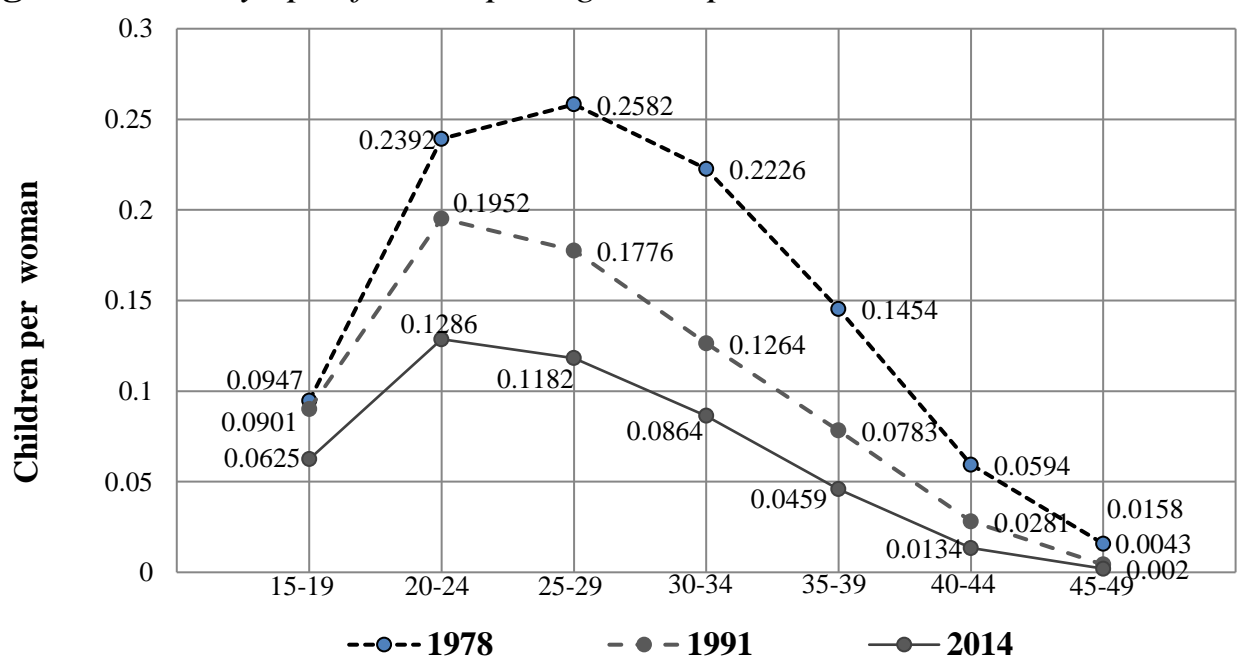

Source: Table 1

But a reduction of fertility specific rates in the country implied that the TFR also fell drastically in the last 40 years. In 1975, according to MFS, the TFR was estimated at 6.03 children per woman, while in 1991 and according to the 1922 NSDD, it was estimated at 3.5 children per woman, which represented a reduction of almost three children in 17 years. However, since then the speed of reduction of TFR in Mexico has decreased. In the last 23 years it only reduced by about one child, from 3.5 in 1991 to 2.29 children per woman in 2014, according to the ICS of 2015 (see Figure 2).

Figure 2. Total Fertility Rate in Mexico, 1975-2014

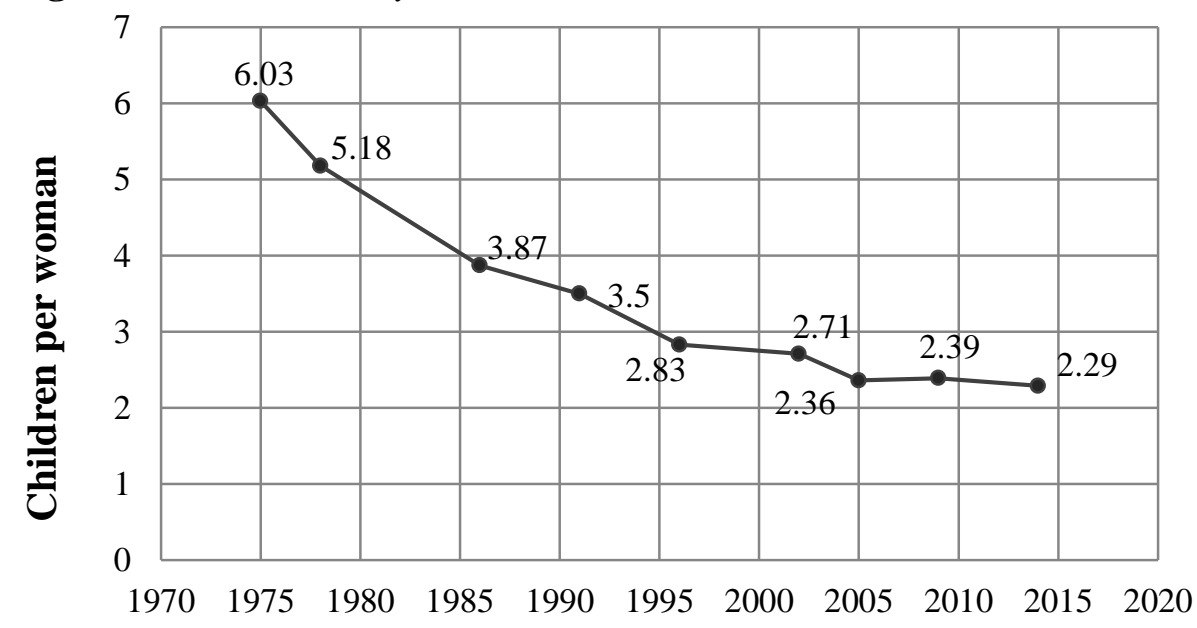

Source:Table 1

\section{Total Fertility Rate Stability}

At first glance, the most recent observations of the last 40 years of the TFR indicate that it has stopped descending with the velocity that was observed earlier 
(see Figure 2). Thus, if the TFR tends to stabilize then its change velocity must be close to zero; therefore, the TFR must not change over time, namely, it must be equal to a constant. This constant is called TFR's stabilizer value, and the answers regarding its existence and the calculation of this constant seem to be in the Stable Bounded Theory (Gonzalez-Rosas 2012).

The Stable Bounded Theory rests in two fundamental postulates. First, in each year the TFR is a random phenomenon, and so according to probability theory, in each year it must have a mean and a variance. Second, the mean of the TFR is equal to a mathematical function, which depends on time, and thus implies by properties of the mean that in each year observations of the TFR will be equal to a quantity determined by the mathematical function plus a certain random deviation, which occurs according to probabilistic law. Medhi (1981) called the mathematical function the deterministic component and the random deviation is called the stochastic component. Under these postulates then, the behavior equations of observations and the mean of the TFR in each time would be:

$$
\begin{aligned}
& \Phi_{t}=f(t)+\varepsilon_{t} \\
& \mu_{\Phi}^{t}=f(t)
\end{aligned}
$$

Where:

$\Phi_{t}$, denotes the observation of the random variable of the TFR in time t, $f(t)$, is a mathematical function unknown, $\varepsilon_{t}$, are random variables that we suppose independents, with distribution law Normal, mean $\mu_{\varepsilon}=0$, constant variance $\sigma_{\varepsilon}^{2}$, and $\mu_{\Phi}^{t}$, denotes the random variable mean TFR in time t.

Due to the change velocity of the TFR between a time $t$ and other $t+1$ is measured with the slope of the straight line that joins two points of a bidimensional space defined by time and the TFR. In order to test the stability hypothesis, we calculated slopes and middle values ${ }^{2}$ of two consecutive TFR values of the following form:

$$
\begin{aligned}
& \nabla_{i}=\frac{\Phi_{i+1}-\Phi_{i}}{t_{i+1}-t_{i}} \\
& V M_{i}=\Phi_{i}+\frac{\Phi_{i+1}-\Phi_{i}}{2}
\end{aligned}
$$

\footnotetext{
${ }^{2}$ The Stable Bounded Theory proves that exist three estimators of stabilizer value. One of them is associated with $y i$, other with $y i+1$, and one more with middle value between the two. This theory also proves that the best of the three is middle value.
} 
Where:

$\nabla_{i}$, denotes slope of straight line between $\left(\Phi_{i}, t_{i}\right)$ and $\left(\Phi_{i+1}, t_{i+1}\right)$ of two dimensional space defined by time and the TFR (Leithold, 1973, p. 137), and

$V M_{i}$, represents middle value between TFR data denoted as $\Phi_{i}$ and $\Phi_{i+1}$.

In Table 2, you can see the results of calculations and in Figure 3 middle values of the TFR are on axis $\mathrm{X}$, and slope values are on axis $\mathrm{Y}$.

Table 2. Total Fertility Rate, Middle Points and Slopes in Mexico 1975-2014

\begin{tabular}{|l|c|c|c|c|}
\hline Year & Time & TFR & Middle Points & Slopes \\
\hline 1975 & 0 & 6.03 & 5.61 & -0.2833 \\
\hline 1978 & 3 & 5.18 & 4.53 & -0.1638 \\
\hline 1986 & 11 & 3.87 & 3.69 & -0.0740 \\
\hline 1991 & 16 & 3.5 & 3.17 & -0.1340 \\
\hline 1996 & 21 & 2.83 & 2.77 & -0.0200 \\
\hline 2002 & 27 & 2.71 & 2.54 & -0.1167 \\
\hline 2005 & 30 & 2.36 & 2.38 & 0.0075 \\
\hline 2009 & 34 & 2.39 & 2.34 & -0.0200 \\
\hline 2014 & 39 & 2.29 & & \\
\hline
\end{tabular}

Source: Table 1 and own calculations based on equations 3 and 4.

As can be seen in Figure 3, the points are not exactly on the straight line, what can be explained if we suppose that slope is also random, and so, under this hypothesis according to the probability theory the slope must have a mean and a variance, what would imply that the points would be the observations of the slope while the straight line would be its mean, mathematically this situation would be able represented as:

$$
\begin{aligned}
& \nabla_{i}=\alpha+\beta \Phi+\omega_{i} \\
& \mu_{\nabla}=\alpha+\beta \Phi .
\end{aligned}
$$

Where:

$\nabla_{i}$, denotes the observation $i$ of the random variable of the slope,

$\Phi$, denotes the TFR,

$\alpha$ and $\beta$, are unknown constants, and

$\omega_{i}$, are random variables that we suppose independents, with distribution law

Normal, mean $\mu_{\omega}=0$, constant variance $\sigma_{\omega}^{2}$. 
Figure 3. Slopes and Middle Points of the Total Fertility Rate in México, 19752014

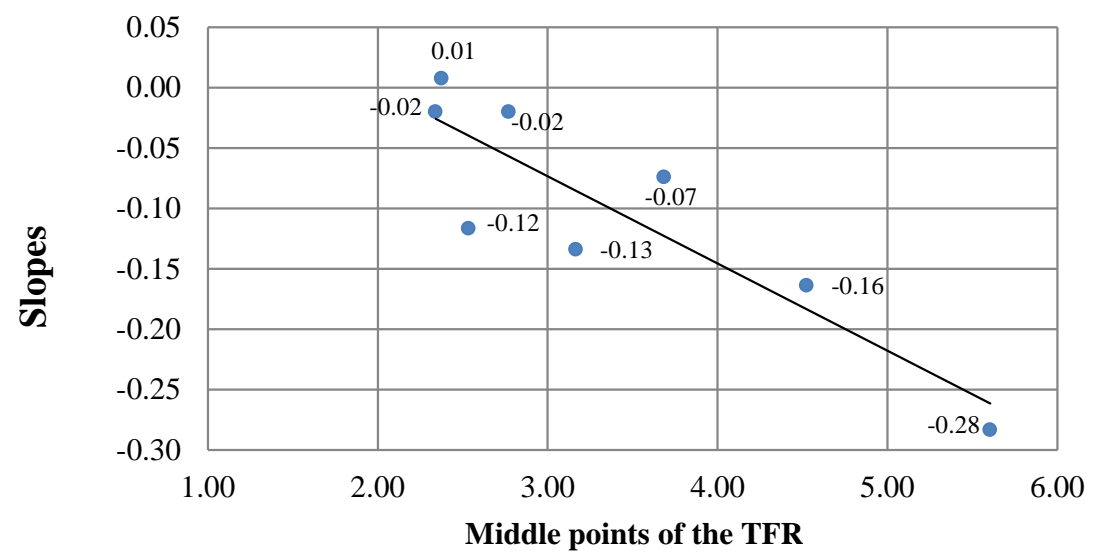

Source: Table 2

You can also see in Figure 3, that when middle values of the TFR are reduced, the points approach zero and also the slope values approach zero. Furthermore, you can also observe that the straight-line intersects axis X near the 2 value, which empirically proves that the stabilizer value of the TFR exists.

From a mathematical point of view, the stabilizing value is equal to the value of the TGF that makes the slope of Equation 6 become zero, that is:

$$
0=\alpha+\beta \Phi
$$

and then, if we do some algebraic operations, we find that the stabilizer value of the TFR denoted as $K$ is:

$$
K=\frac{-\alpha}{\beta}
$$

This result indicates that to calculate the stabilizer value of the TFR, it is necessary to calculate the constants $\alpha$ and $\beta$ of Equation 6 . To estimate these constants, a simple linear regression model was fitted to the data of Figure 3 . The following table presents the ordinary least squares estimates of the $\alpha$ and $\beta$ parameters and the p-values to determine their statistical significance.

Table 3. Parameters Estimate of the Equation 6 and p-values to prove its Statistical Significance

\begin{tabular}{|l|c|c|c|c|}
\hline Parameter & Estimate & $\begin{array}{c}\text { Standard } \\
\text { error }\end{array}$ & t-value & p-value \\
\hline$\alpha$ & 0.1407 & 0.0571 & 2.46 & 0.049 \\
\hline$\beta$ & -0.0709 & 0.0101 & -4.41 & 0.005 \\
\hline
\end{tabular}

Source: Own calculations based on middle points and slopes of table 2 . 
As can be seen, the two coefficients are significantly different from zero, so that to estimate the stabilizer value of the TFR, the estimations of the coefficients $\alpha$ and $\beta$ were substituted in 7, thus obtaining the conclusion that TFR in Mexico will stabilize with the value:

$$
\begin{aligned}
& K=\frac{-0.1407}{-0.0709} \\
& K=1.98
\end{aligned}
$$

In addition to the significance of the parameters, the p-value of the $F$ statistic was 0.0045 , which proves that the straight-line assumption in 6 is true, and the coefficient of determination was $76.39 \%{ }^{3}$. These results prove mathematically the existence of the stability of the TFR. A parabolic model also was fitted to the same data, but the coefficient of the quadratic term was not significant, which proves then there are no two stabilizing values for the TFR of Mexico.

Finally, it is important to clarify that the value $K=1.98$ is a bound for the mean of the TGF but not for the observations, which according to the theory of probability will deviate a certain amount around the mean depending on its variance. Therefore they can be greater or less than $\mathrm{K}=1.98$, but their occurrence will be governed by a probabilistic law.

\section{The Equation of the TFR and Time}

According to the postulates of the Stable Bounded Theory, the behavior equations of the observations and mean of the TFR in each time are:

$$
\begin{aligned}
& \Phi_{t}=f(t)+\varepsilon_{t} \\
& \mu_{\Phi}^{t}=f(t)
\end{aligned}
$$

The problem is that in practice the stabilizer function $f(t)$ is unknown. However, the trend of the data and the existence of the stabilizer value can give us an idea of what its derivative is, and the theory of differential equations can help us to deduce its mathematical equation. Firstly, according to trends of observed data, the function has to be decreasing, and so, its derivative will be negative. Secondly, due to the existence of a stabilizer value, its derivative will have to be zero in the stabilizer value. Based on these properties, the Stable Bounded Theory deduces a function that satisfies the properties mentioned.

The Stable Bounded Theory supposes that the derivative of the unknown function is given by a product of two functions, $h_{1}(\Phi)$ and $h_{2}(t)$, one that depends on the TFR and other that depends on time. These form a differential equation of separable variables (Wilye 1979), which has as a solution a function

\footnotetext{
${ }^{3}$ The residual analysis indicates that the random variables of the model are distributed normal, are independent and have constant variance
} 
relating the TFR and time, namely:

$$
\frac{d \Phi}{d t}=h_{1}(\Phi) h_{2}(t)
$$

Now, since the derivative must be negative and equal to zero in the stabilizer value $\mathrm{K}$, the functions $h_{1}(\Phi)$ and $h_{2}(t)$ can be as follows:

$$
\begin{aligned}
& h_{1}(\Phi)=(\Phi-K) \\
& h_{2}(t)=m \\
& \frac{d \Phi}{d t}=(\Phi-K) m
\end{aligned}
$$

Where $m$ is a constant less than zero and $\mathrm{K}$ is the stabilizer value.

We can observe that due to $K$ being an inferior bound of the TFR, then quantity $(\Phi-K)$ is always positive, but when you multiply by $m$, then $(\Phi-K)$ $m$ is negative. This derivative is negative as we require. On the other hand, when the TFR is equal to $\mathrm{K}$ then the derivative is zero, another condition we require.

Now separating variables we have:

$$
\int \frac{1}{(\Phi-K)} d \Phi=\int m d t
$$

Solving the indefinite integrals, we arrive at:

$$
\ln (\Phi-K)=m t+C
$$

Finally, clearing the variable $\Phi$ we obtain:

$$
\Phi_{t}=\left(\lambda e^{m t}\right)+K \quad ; \operatorname{con} m<0
$$

Where $\Phi_{t}$ denotes the TFR at time $t, K$ the stabilizer value, $e$ is the exponential function, and $\lambda$ and $m$ are unknown constants, such that $\lambda$ defines the initial conditions of the TFR at time zero and $m$ represents the amount of reduction per unit time. Since the parameters $\lambda$ and $m$ determine how quickly the TFR approaches stability, they are called the parameters of speed.

We can observe that since $m$ is negative, Equation 10 is decreasing; further, when $t$ tends infinite, $\lambda e^{m t}$ tends zero, so that the TFR tends towards $\mathrm{K}$, the stabilizer value. Equation 10 is called the decreasing exponential function and solves theoretically the problem of existence of a stabilizer function of behavior equations, both of observations as the mean of the TFR. 


\section{Estimation of the Parameters of the Speed}

According to Draper and Smith (1966), Equation 10 is not linear at parameters $\lambda, m$ and $K$, so they cannot be estimated by the method of least squares. However, if at Equation 10 we pass $\mathrm{K}$ to the left of equality and if we apply the natural logarithm at both sides of the equation, we obtain:

$$
\ln (\Phi-K)=\ln \lambda+m t
$$

That is, the result is a linear equation at the parameters $\ln \lambda$ and $m$, which can be estimated by the method of ordinary least squares or generalized least squares. This suggests that the estimation of the parameters of Equation 10, can be done in two stages. First, we estimate $K$ and then we estimate $\ln \lambda$ and $m^{4}$. The variable $\ln (\Phi-K)$ is called the transformed of the TFR.

Table 4. Year, Time, TFR and Transformed of the TFR in Mexico

\begin{tabular}{|l|c|c|c|}
\hline Year & Time & TFR & Transformed of the TFR \\
\hline 1975 & 0 & 6.03 & 1.3987 \\
\hline 1978 & 3 & 5.18 & 1.1632 \\
\hline 1986 & 11 & 3.87 & 0.6366 \\
\hline 1991 & 16 & 3.5 & 0.4187 \\
\hline 1996 & 21 & 2.83 & -0.1625 \\
\hline 2002 & 27 & 2.71 & -0.3147 \\
\hline 2005 & 30 & 2.36 & -0.9676 \\
\hline 2009 & 34 & 2.39 & -0.8916 \\
\hline 2014 & 39 & 2.29 & -1.1712 \\
\hline
\end{tabular}

Source: Time was calculated as year-1975; Transformed was calculated based on equation 11.

Replacing the value $\mathrm{K}=1.98$ in Equation 11, we calculated the transformed of the TFR (see Table 4). In Figure 4 we can check that the relation between the transformed of the TFR and time in Mexico is given effectively by a straight line, as is predicted by the Stable Bounded Theory. Thus, it is linear at parameters $\ln \lambda$ and $m$. In order to estimate them, we adjusted a simple regression model to data of Figure 4.

\footnotetext{
${ }^{4}$ The residual analysis indicates that the random variables of the model are distributed normal, are independent and have constant variance.
} 
Figure 4. Transformed of the TFR and time in Mexico, 1975-2014

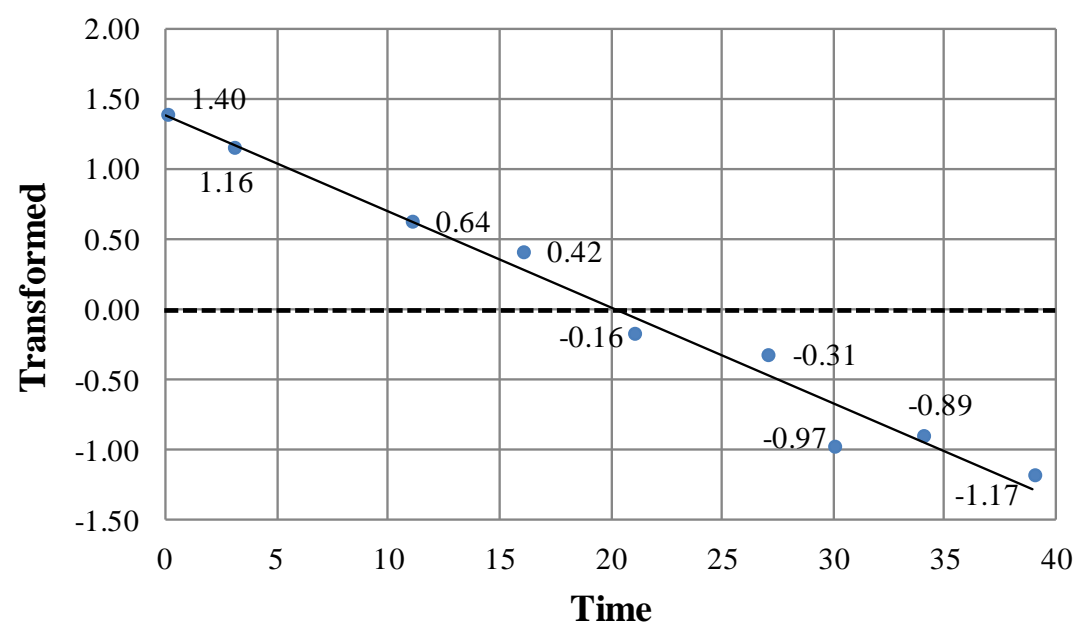

Source: Table 4

Table 5 presents the ordinary least squares estimates of the parameters and the p-values that prove their statistical significance. Note that both parameters are statistically significant with values $\ln \lambda=1.386$ and $m=-0.0683$. To obtain the estimation of the $\lambda$, we apply an exponential function to 1.386 obtaining $\lambda=3.999$.

Table 5. Parameters Estimation of the Equation 11 and p-values to prove its Statistical Significance

\begin{tabular}{|l|c|c|c|c|}
\hline Parameter & Estimation & $\begin{array}{c}\text { Standard } \\
\text { error }\end{array}$ & t-value & p-value \\
\hline $\ln \lambda$ & 1.386 & 0.0932 & 14.87 & 0.001 \\
\hline$M$ & -0.0683 & 0.0039 & -17.49 & 0.001 \\
\hline
\end{tabular}

Source: own calculations based on table 4 .

With the estimation of the parameters of speed, the equations of behavior of the observations and of the average of the TFR as a function of time were completely solved. The equation of the observations can be used to elaborate interval projections of the TFR, whereas the equation of the mean can be used to carry out point projections of the mean of the TFR.

\section{Results}

\section{Punctual Forecasts of the TFR in Mexico}

The results above prove that behavior of the mean of the TFR through time is governed by following mathematical equation:

$$
\mu_{\Phi}^{t}=3.999 e^{-0.0683 t}+1.98
$$


Where

$\mu_{\Phi}^{t}$, denotes the mean of the TFR at time $t$,

The constants 3.999 and -0.0683 determine the initial conditions of the mean of the TFR at time zero and the amount of reduction of the mean of TFR per unit time, respectively.

The constant 1.98 represents the stabilizer value of the mean of TFR.

Figure 5. Total Fertility Rate Observed and Estimated in Mexico, 2015-2050

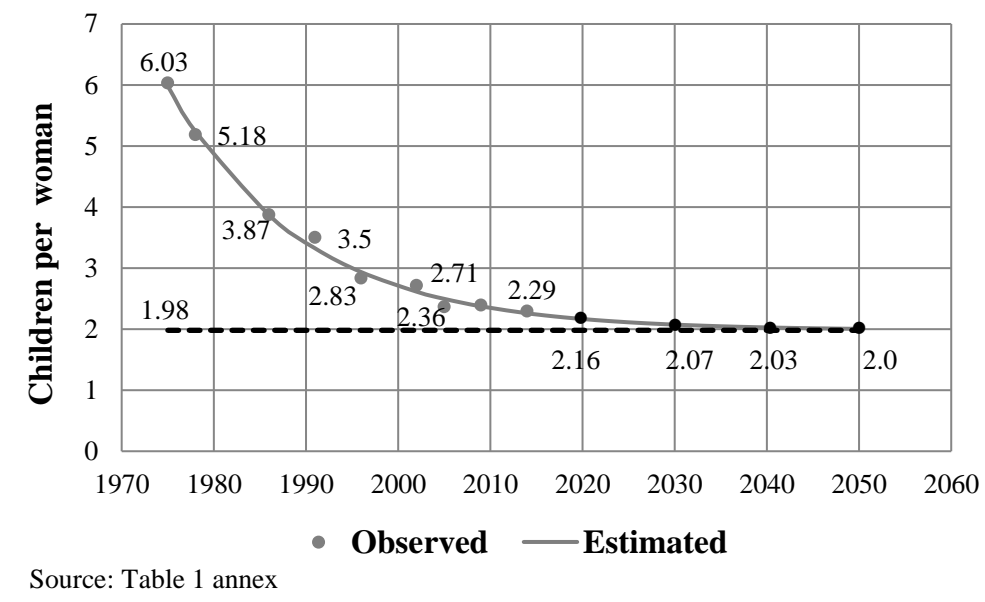

Giving values to the time variable in Equation 12, we obtained punctual forecasts of the mean of the TFR in Mexico for the period 2015-2050. In Figure 5, you can observe that the model is adjusted very well to the observed data, and when time is increased the mean of the TFR approaches the stabilizer value. According to the results of Model 12, we found that in 2020 the mean of TFR will be 2.16 children per woman; in 2030 it will be 2.07 ; in 2040 we expect that it will be 2.03; and finally, in 2050 it will have reached stability arriving at 2.0 children per woman.

\section{Discussion}

When we analyze TFR's data through time we realized us that they had a irregular behavior but with a decreasing trend, such that, when we consider that in each moment of time the TFR rate is a random phenomenon, then we could explain behavior irregular observed of the rate. However, this hypothesis implied that we could not forecast the total fertility rate, since random phenomena cannot be predicted. But if the objective of our paper was predict the TFR, so the question arose, how can we predict something that is unpredictable.

The answer comes out of probability theory. According to this theory, the total fertility rate must have a mean and a variance, so that, when we assumed the mean had a deterministic behavior given by a mathematical function dependent on time, then we would be able predict at least the mean of the total fertility rate. According to the trend of data, the function had to be decreasing, however fertility 
cannot get down until arrives to zero, since evidence of some developed countries indicate that is going to stabilize, this situation brought us two more questions. Firstly, what is the value where the total fertility rate is going to stabilize in future? And secondly, what is the function that it must to use to predict the total fertility rate?

These two questions were answered using the Stable Bounded Theory, which allowed us to prove the existence of a stabilizer value and to calculate it. Also, we found the function that allowed us to do the predictions of the total fertility rate.

Finally, we achieved the surprising result that the stabilizing function was not a logistic function, as in many studies. It can be verified by having data for when the rate begins to lower slowly, then when it lowers rapidly, and finally when it returns to lowering slowly. Unfortunately, in this exercise we only had data of when the rate drops rapidly and when it falls slowly, which can be a limitation.

\section{Conclusions}

In Mexico, for the period 1975-2015, the behavior of the mean of the total fertility rate through time is governed by a mathematical function that depends on time.

The function is determined by three parameters, the stabilizer value $K=1.98$ and the quickness parameters $\lambda=3.999$ and $m=-0.0683$. These parameters can change according to the country or analyzed period.

This model cannot be used to know the behavior of total fertility rate in the past, because according to this model when we go back in time, the total fertility rate grows and grows, which is not possible.

In Mexico for explaining the evolution of the total fertility rate through time, the demographers have used a logistic function. However, this paper proves that this is an error, since two stabilizer values do not exist as the logistic function needs.

Although this exercise was done with data from Mexico, it is important to make it clear that the Stable Bounded Theory can be applied to any country where data on the total fertility rate are available.

It is necessary to warn that the results of this paper are based on the assumption that the social, economic and political conditions will continue without change. If this assumption it is not fulfilled, the forecasts will not be true.

Also, it is necessary to warn that the mathematical modeling of reality is based on many assumptions, and the theoretical results are true only if the assumptions are fulfilled. Thus, it is necessary to expend great effort to prove that the assumptions are true.

Finally, any exercise to predict the future is exposed to a lot of error sources: wrong data, false assumptions and hypotheses, incorrect models, and so on. Therefore, it is necessary to identify all possible error sources, and then utilize methodologies that minimize those errors. The Stable Bounded Theory is an example of that. 


\section{References}

Alkema L, Raftery AE, Gerland P, Clark SJ, Pelletier F, Buettner F, Heilig GK (2011) Probabilistic projections of the total fertility rate for all countries. Demography 48(3): 815-839. Retrieved from https://bit.ly/2Hj1p33. [Accessed 9 April 2017]. doi: 10.1007/s13524-011-0040-5.

Alkema L, Gerland P, Raftery A, Wilmoth J (2015) The United Nations Probabilistic Projections: An introduction to demographic forecasting with uncertainly. Foresight (Colchester, Vt), 2015(37): 19-24. Retrieved from https://bit.ly/2qTsoHu. [Accessed 10 April 2017].

Ashraf QH, Weil DN, Wilde J (2013) The Effect of fertility reduction on economic growth. In Population and Development Review 39(1): 97-130. doi:10.1111/j.17 28-4457.2013.00575.x

Coordinación del Programa Nacional de Planificación Familiar (NSPUCM) (1979) Encuesta Nacional de Prevalencia en el Uso de Métodos Anticonceptivos. Resultados nacionales [Survey of Prevalence in the Use of Contraceptive Methods. National results]. México, p. 219.

Dirección General de Planificación Familiar (1989) Encuesta Nacional sobre Fecundidad y Salud 1987 [National Survey on Fertility and Health 1987]. Mexico Ministry of Health. Undersecretariat of Health Services, p. 231.

Draper J, Smith, W (1966) Applied Regression Analysis. New York: John Wiley \& Sons.

González-Rosas J (2012) La Teoría Estable Acotada: Fundamentos, conceptos y métodos, para proyectar los fenómenos que no pueden crecer o decrecer indefinidamente [The Stable Bounded Theory: Fundamentals, concepts and methods, to project phenomena that cannot grow or decrease indefinitely]. Saarbrucken, Germany: Spanish Academic editorial.

INEGI (2017). Principales resultados de la Encuesta Intercensal de 2015 [Main results of the 2015 Intercensal Survey]. Retrieved from https://bit.ly/2qPjpYW. [Accessed 18April 2017].

Leithold L (1973) El Cálculo: Con geometría analítica [The calculation with analytic geometry], $2^{\text {nd }}$ ed. Mexico: Harla S.A. Of C.V.

Lutz W (2006) Fertility rates and future populations trends: will Europe's birth rate recover or continue decline? International Journal of Andrology. Retrieved from https://bit.ly/2HoXair. [Accessed 11 April 2017]. doi: 10.1111/j.1365-2605.2005. 00639x.

Medhi J (1981) Stochastic Processes, $2^{\text {nd }}$ ed. New York: John Wiley \& Sons.

Partida-Bush V (2008) Proyecciones de la población de México, de las entidades federativas, de los municipios y de las localidades 2005-2050 [Projections of the population of Mexico, the states, municipalities and localities 2005-2050]. Mexico: Methodological document. National Population Council.

Secretaría de Programación y Presupuesto (1979) Encuesta Mexicana de Fecundidad. Primer informe nacional [Mexican Fertility Survey. First national report]. Mexico, p. 239.

Sobotka T, Zeman K, Basten S (2015) The Low Fertility Future? Projections Based on Different Methods Suggest Long-term Persistence of Low Fertility. Retrieved from https://bit.ly/2HjldDH.

United Nations, Department of Economic and Social Affairs, Population Division (2015). World Fertility Patterns 2015. Data Booklet (ST/ESA/ SER.A/370). Retrieved from https://bit.ly/1SvRZyO. [Accessed 10 April 2017].

Wilye CR (1979) Differential equations. Mexico: McGraw Hill. 


\section{Annex}

Table 1. Total Fertility Rate Projections in Mexico, 2015-2050

\begin{tabular}{|l|c|c|c|c|c|}
\hline Year & Time & Total Fertility Rate & Year & Time & Total Fertility Rate \\
\hline 2015 & 40 & 2.24 & 2033 & 58 & 2.06 \\
\hline 2016 & 41 & 2.22 & 2034 & 59 & 2.05 \\
\hline 2017 & 42 & 2.21 & 2035 & 60 & 2.05 \\
\hline 2018 & 43 & 2.19 & 2036 & 61 & 2.04 \\
\hline 2019 & 44 & 2.18 & 2037 & 62 & 2.04 \\
\hline 2020 & 45 & 2.16 & 2038 & 63 & 2.03 \\
\hline 2021 & 46 & 2.15 & 2039 & 64 & 2.03 \\
\hline 2022 & 47 & 2.14 & 2040 & 65 & 2.03 \\
\hline 2023 & 48 & 2.13 & 2041 & 66 & 2.02 \\
\hline 2024 & 49 & 2.12 & 2042 & 67 & 2.02 \\
\hline 2025 & 50 & 2.11 & 2043 & 68 & 2.02 \\
\hline 2026 & 51 & 2.10 & 2044 & 69 & 2.02 \\
\hline 2027 & 52 & 2.09 & 2045 & 70 & 2.01 \\
\hline 2028 & 53 & 2.09 & 2046 & 71 & 2.01 \\
\hline 2029 & 54 & 2.08 & 2047 & 72 & 2.01 \\
\hline 2030 & 55 & 2.07 & 2048 & 73 & 2.01 \\
\hline 2031 & 56 & 2.07 & 2049 & 74 & 2.01 \\
\hline 2032 & 57 & 2.06 & 2050 & 75 & 2.00 \\
\hline
\end{tabular}

Source: Own calculations based on equation 12 